\title{
Semi-Quantitative Solid-State NMR Study of the adsorption of Soybean Oils on Silica and Its Significance for Rubber Processing
}

\author{
Chuanyu Yan, ${ }^{\dagger \neq}$ Arpan Datta Sarma, ${ }^{\dagger, \ddagger}$ Enzo Moretto, ${ }^{\dagger, \ddagger}$ Jean-Sébastien Thomann, ${ }^{\dagger}$ Pierre \\ Verge ${ }^{\dagger}$, Daniel Schmidt, ${ }^{\dagger}$ François Kayser ${ }^{\S}$ and Reiner Dieden ${ }^{*},{ }^{\dagger}$ \\ † Department of "Materials Research and Technology", Luxembourg Institute of Science and Technology, \\ Avenue des Hauts-Fourneaux, L-4362 Esch-sur-Alzette, Luxembourg. \\ $\ddagger$ Faculty of Science, Technology and Medicine, University of Luxembourg, Avenue des Hauts-Fourneaux, \\ L-4365 Esch-sur-Alzette, Luxembourg. \\ § Goodyear Innovation Center Luxembourg, Avenue Gordon Smith, L-7750 Colmar Berg, Luxembourg \\ *Correspondence: reiner.dieden@list.lu; Tel: (+352) 2758884576
}

\section{Table of content}

Figure S1: Supplementary information about the deuterated silica.

Figure S2-S3: Supplementary information for the set-up of the internal mixer where the mixing

Figure S4: The optimization process of ${ }^{29} \mathrm{Si} \mathrm{MCP}$ experiments.

Figure S5-S7 and Table S1: Supplementary liquid-state NMR data for the characterization of SBOs.

Figure S8-S9 and Table S2: Supplementary solid-state NMR data for characterization of SBOsadsorbed silicas.

Figure S10: The physical appearance of the model rubber compounds.

Figure S11: Supplementary solid-state NMR data for characterization of model rubber compounds: a comparison between regular silica and deuterated silica. 


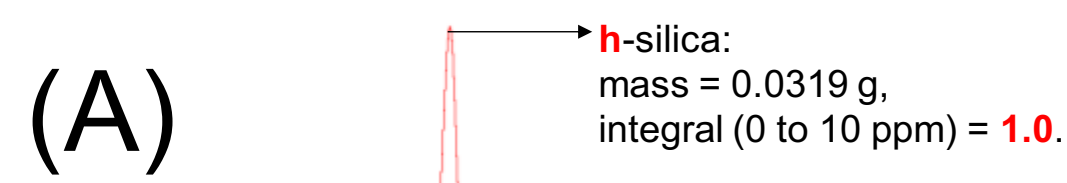

${ }^{1} \mathrm{H}$ one-pulse ssNMR spectra:

MAS $=8 \mathrm{kHz}$,

$\mathrm{NS}=16$,

$R G=2$,

$\mathrm{RD}=6$ seconds.

d-silica:

mass $=0.0327 \mathrm{~g}$,

integral $(0$ to $10 \mathrm{ppm})=\mathbf{0 . 3 8}$

(relative to h-silica).

$\mathrm{H}^{10}$ Chemical Shift $(\mathrm{ppm})$
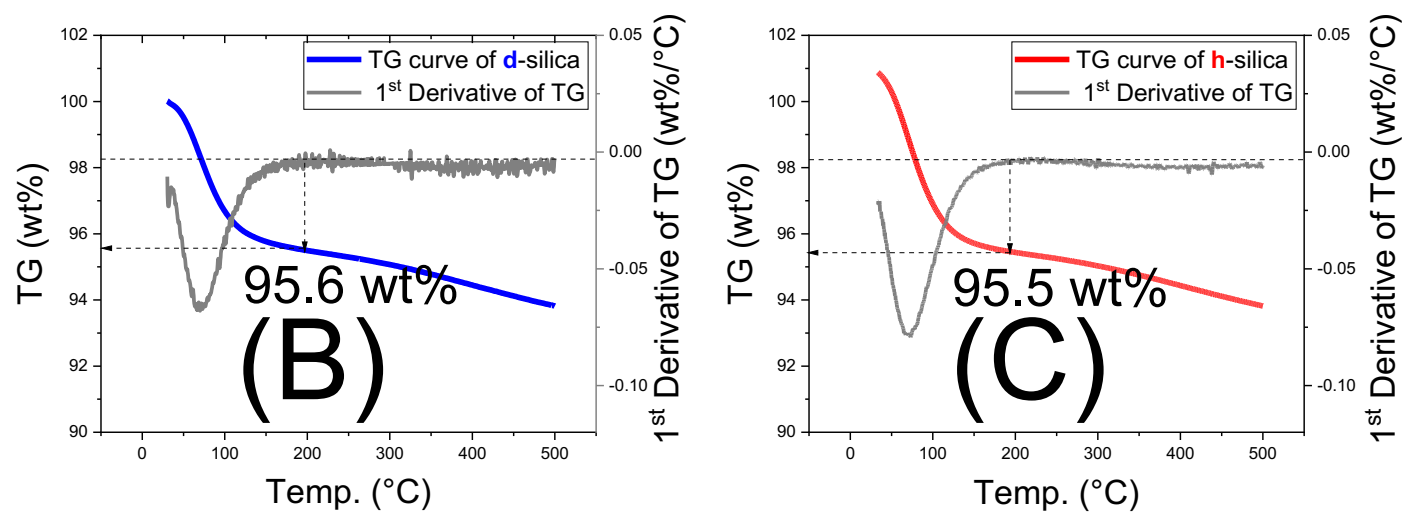

Figure S1. Estimation of the deuteration degree of silica. (A) Quantitative ${ }^{1} \mathrm{H}$ one-pulse ssNMR spectra of silica before and after deuteration. (Please refer to the Experimental Section for more information about the NMR spectrometer.) In brief, the two spectra were acquired using the same parameters: magic angle spinning rate (MAS $=8 \mathrm{kHz}$ ), number of accumulated scans (NS $=16)$, receiver gain $(R G=2)$ and recycle delay $(R D=6 s)$. In order to ensure all the protons are fully relaxed to equilibrium (i.e., being quantitative), more spectra (not shown here) were also acquired at RD $=8$ and 10 seconds, which yielded identical spectra. (B)\&(C) Thermogravimetric analysis (TGA) of the silica before and after deuteration. TGA curves were acquired on a Mettler Toledo-TGA 2 instrument, heating from RT to $500^{\circ} \mathrm{C}$ in alumina crucibles at $20^{\circ} \mathrm{C} / \mathrm{min}$ in a nitrogen atmosphere. The moisture $\left(\mathrm{H}_{2} \mathrm{O} / \mathrm{D}_{2} \mathrm{O}\right)$ content was estimated by the mass loss at $\sim 190{ }^{\circ} \mathrm{C}$, which was indicated by the first plateau (dehydration stage) of the first order derivative of the TGA curve. In this case, the moisture contents were 4.4 and $4.5 \mathrm{wt} \%$ before and after deuteration, respectively, which were effectively identical. Note that the starting wt $\%$ of h-silica was slightly higher than $100 \%$, likely due to the uptake of environmental moisture of the sample in the TGA pan prior to initiation of the analysis. However, this did not change the analytical results given that the initial mass was determined prior to any such moisture uptake. 


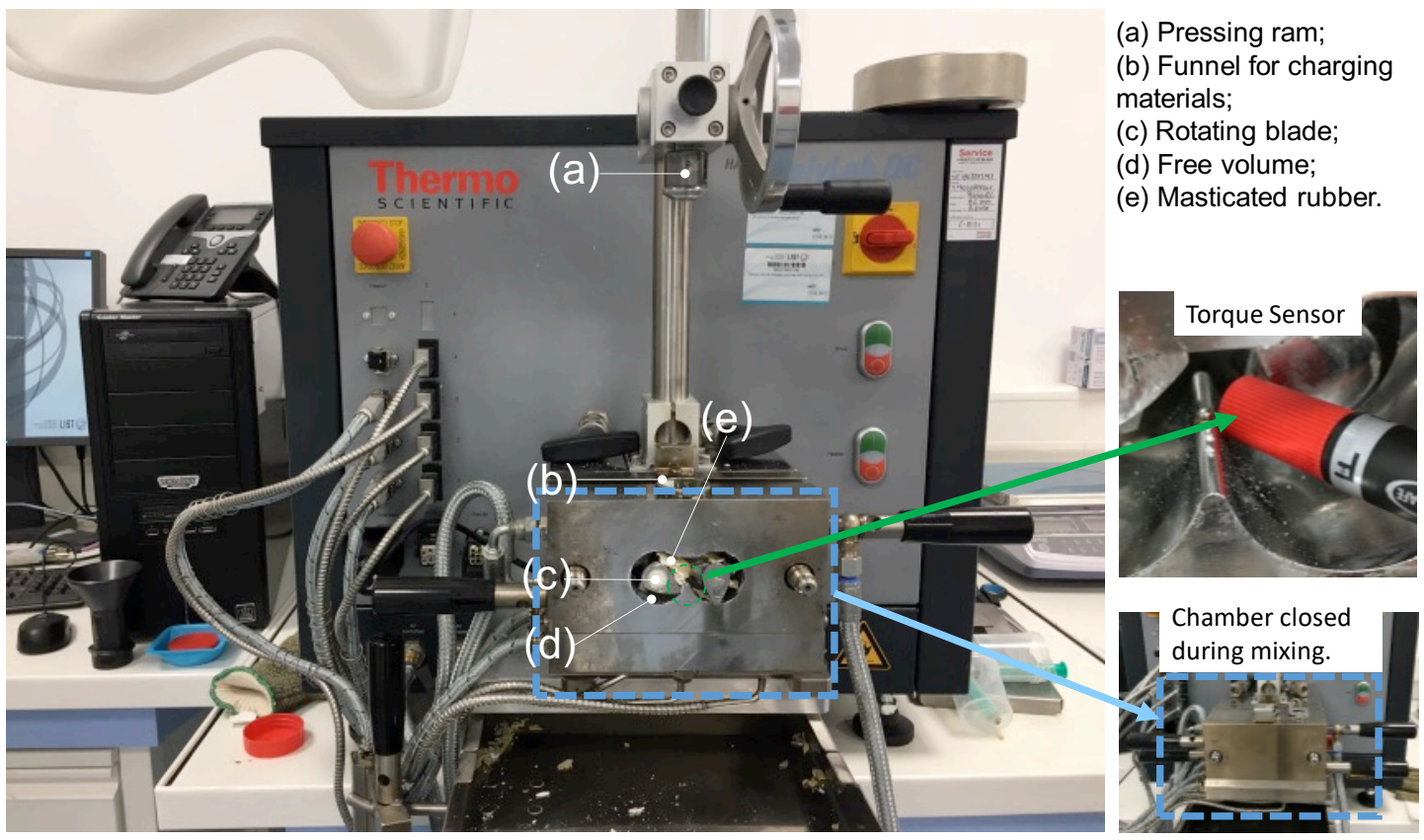

Figure S2. The structure of the HAAKE ${ }^{\mathrm{TM}}$ PolyLab ${ }^{\mathrm{TM}} \mathrm{QC}$ internal mixer (Thermo Scientific) used in this work.

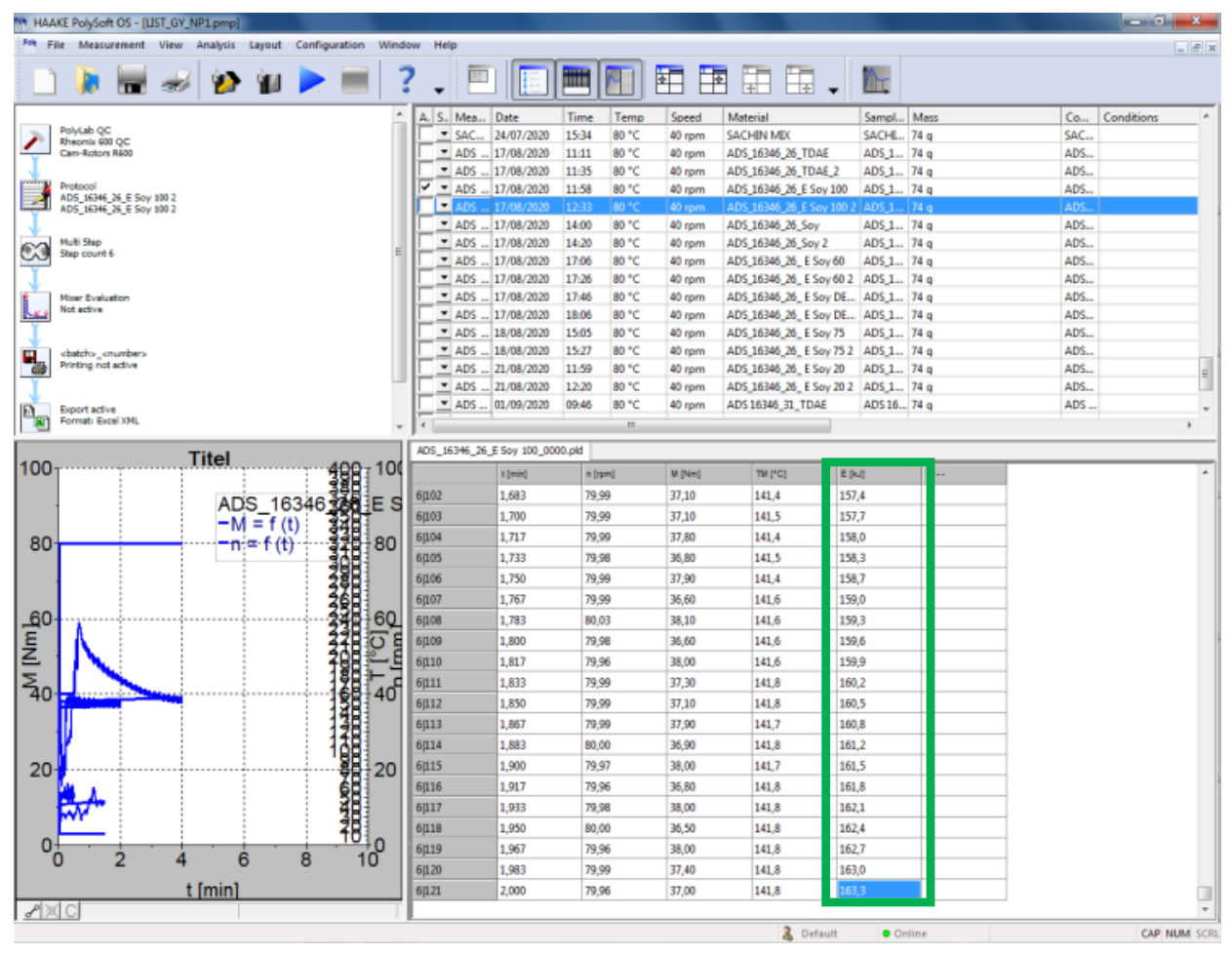

Figure S3. Mixing energy as recorded by the control program. Note that the final mixing energy is the accumulated energy throughout the multiple-stage mixing process. 


\section{Supporting Information}
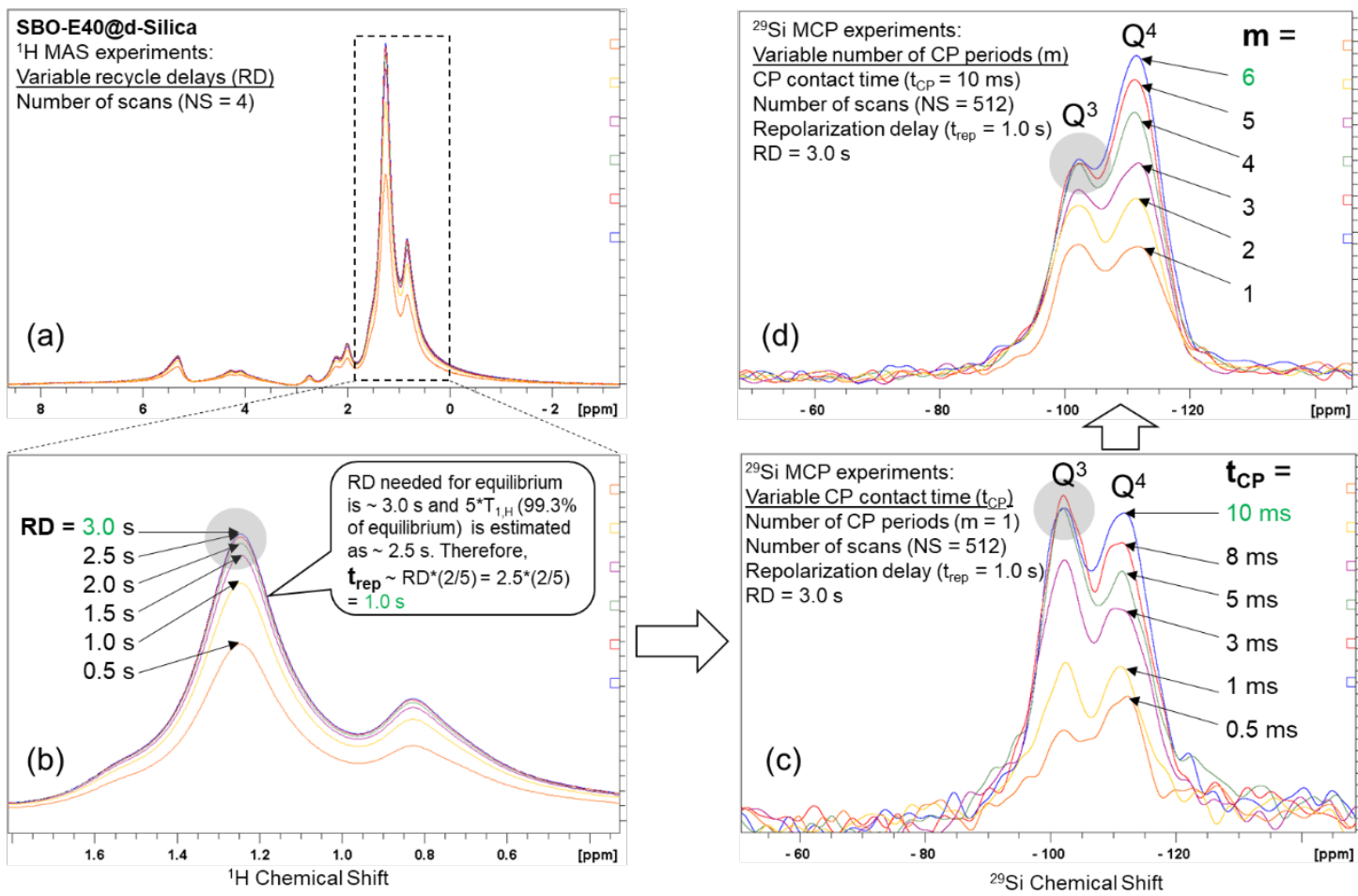

Figure S4. The optimization process of parameters for ${ }^{29}$ Si MCP using the SBO-E40@d-Silica. (a\&b) The recycle and repolarization delays $\left(R D\right.$ and $\left.t_{r e p}\right)$ are optimized through a series of ${ }^{1} \mathrm{H}$ one-pulse experiments with variable RD. The $\mathrm{RD}$ needed for returning the protons to thermal equilibrium is around 3 seconds and the $5^{\star} T_{1, \mathrm{H}}($ i.e. $99.3 \%)$ is around 2.5 seconds, so the $t_{\text {rep }}$ is set at 1.0 second. Note that a more accurate measurement of $T_{1, H}$ can be done by the standard saturation recovery or inversion recovery experiments, if needed. (c) The contact time ( $\mathrm{t}_{\mathrm{CP}}$ ) for single cross polarization (CP) step is optimized through a set of ${ }^{29} \mathrm{Si} \mathrm{MCP}$ experiments where the number of CP periods $(\mathrm{m})$ is fixed at 1 . It is found that the $Q^{3}$ intensity reaches a maximum value at $t_{C P}=5$ milliseconds and above, while the $Q^{4}$ intensity continues to increase up to 10 milliseconds (an upper safety limit of our NMR probe for preventing it from over-heating). Therefore, $t_{C P}=10$ milliseconds is chosen in this study. (d) The number of CP periods $(m)$ is also optimized through a set of ${ }^{29} \mathrm{Si}$ MCP experiments where $t_{C P}$ is fixed at 10 milliseconds. It is found that the $\mathrm{Q}^{3}$ intensity reaches a maximum value at $m=4$ and above, while the $Q^{4}$ intensity continues to increase with no immediate sign of reaching a maximum. Since the $Q^{3}$ is the main group responsible for hydrogen bonding with $S B O s$, a maximized $Q^{3}$ is preferred and therefore $m=6$ is chosen in this study. 


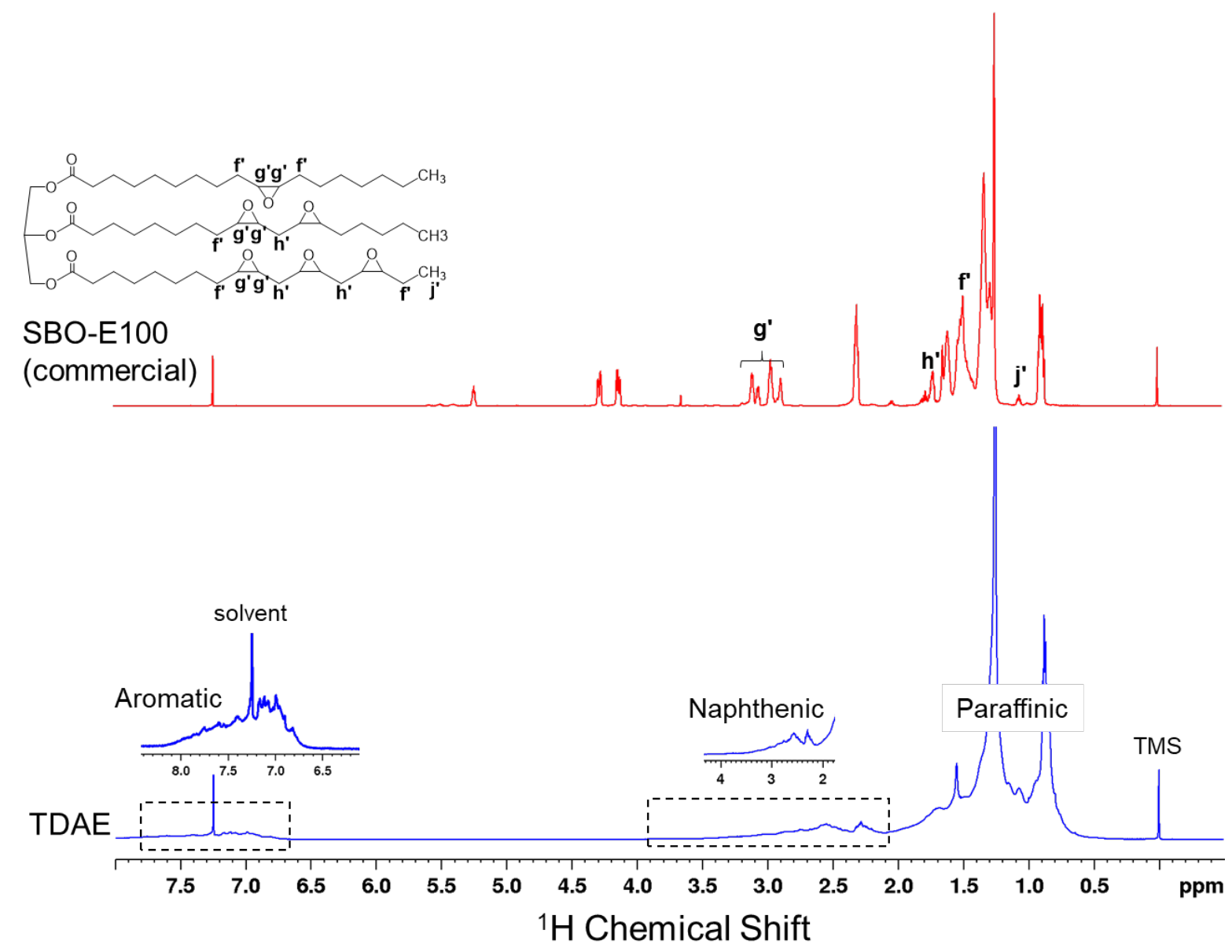

Figure S5. The ${ }^{1} \mathrm{H}$ liquid-state NMR spectra of commercial epoxidized SBO oil and TDAE. 

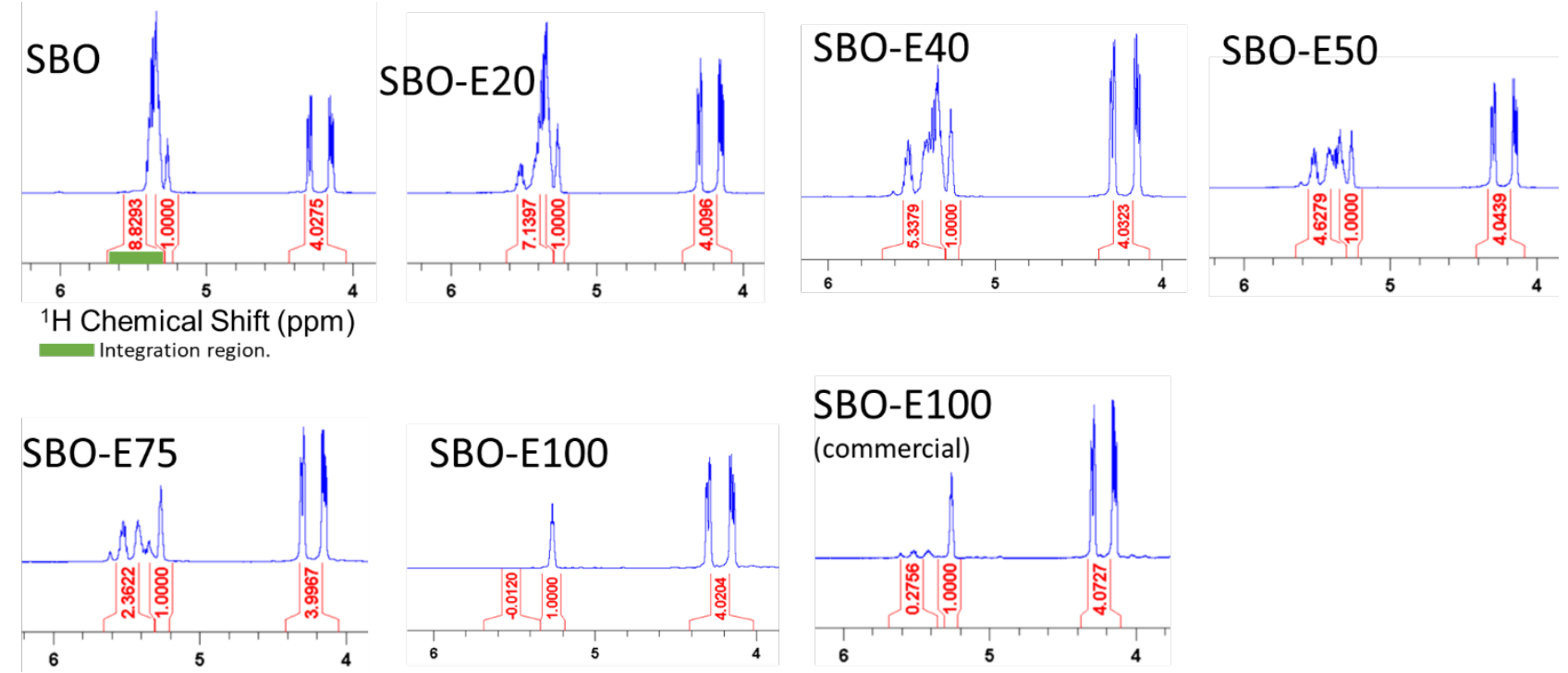

Figure S6. ${ }^{1} \mathrm{H}$ peak integrations in the zoom-in regions of Figure 1 and Figure S5.

Table S1. The exact degree of epoxidation for the SBOs (integrals from Figure S5\&S6).

\begin{tabular}{|c|c|}
\hline Oils & Exact degree of epoxidation \\
\hline SBO & $(8.83-8.83) / 8.83^{*} 100 \%=0 \%$ \\
\hline SBO-E20 & $(8.83-7.14) / 8.83^{*} 100 \%=19 \%$ \\
\hline SBO-E40 & $(8.83-5.34) / 8.83^{*} 100 \%=40 \%$ \\
\hline SBO-E50 & $(8.83-4.63) / 8.83^{*} 100 \%=48 \%$ \\
\hline SBO-E75 & $(8.83-2.36) / 8.83^{*} 100 \%=73 \%$ \\
\hline SBO-E100 & $(8.83-0.01) / 8.83^{*} 100 \%=100 \%$ \\
\hline SBO-E100 (commercial) & $\left(8.34^{\mathrm{a}}-0.28\right) / 8.34^{2 *} 100 \%=97 \%$ \\
\hline
\end{tabular}

aNote: The original $\mathrm{C}=\mathrm{C}$ content was calculated by summing the integrals of peaks $\mathbf{g}$ and $\mathbf{g}$ ' (Figure S5). 


\section{Supporting Information}
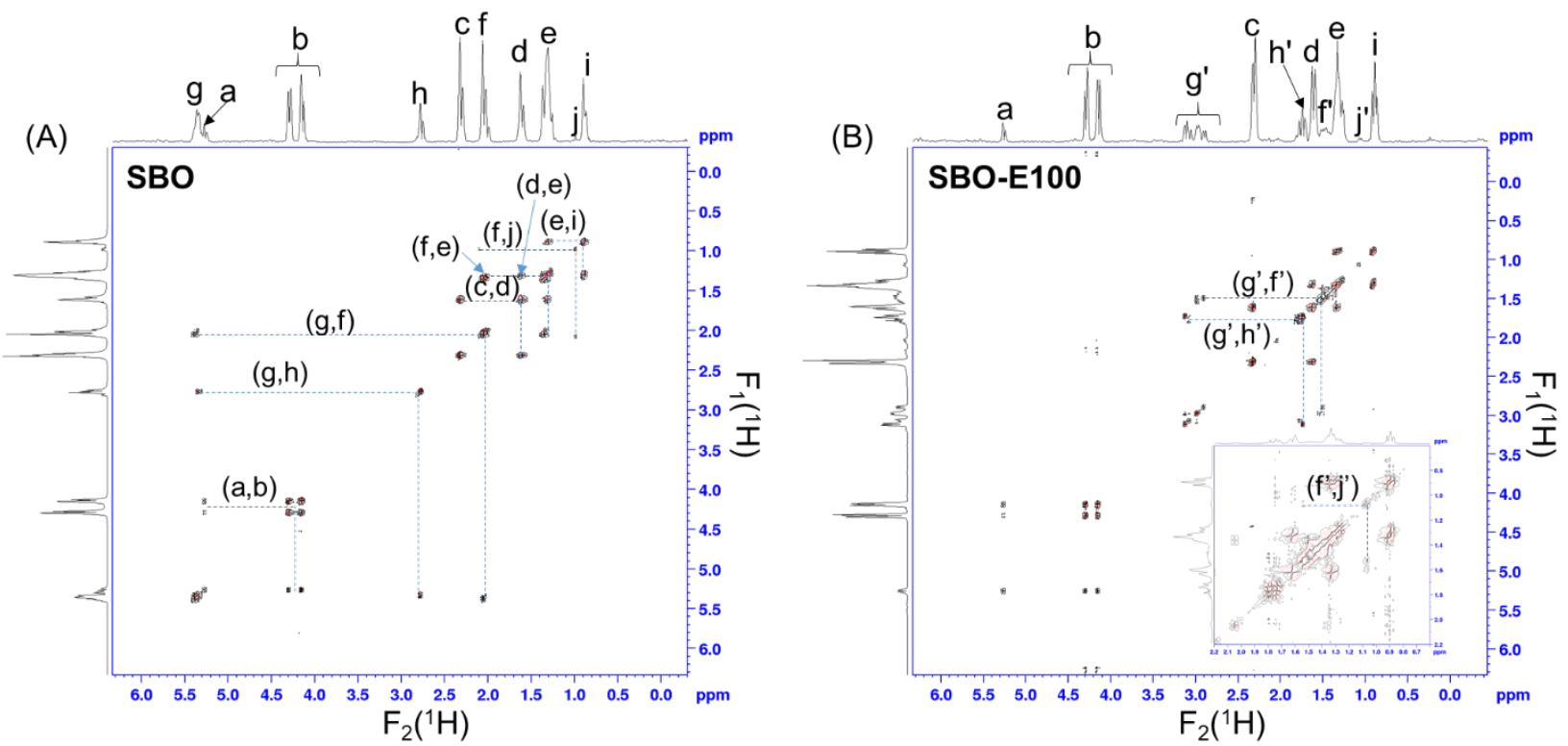

Figure S7. ${ }^{1} \mathrm{H}-{ }^{-1} \mathrm{H}$ COSY-DQF (mixing time $=1.2 \mathrm{~ms}$ ) spectra of SBO and SBO-E100. 


\section{Supporting Information}
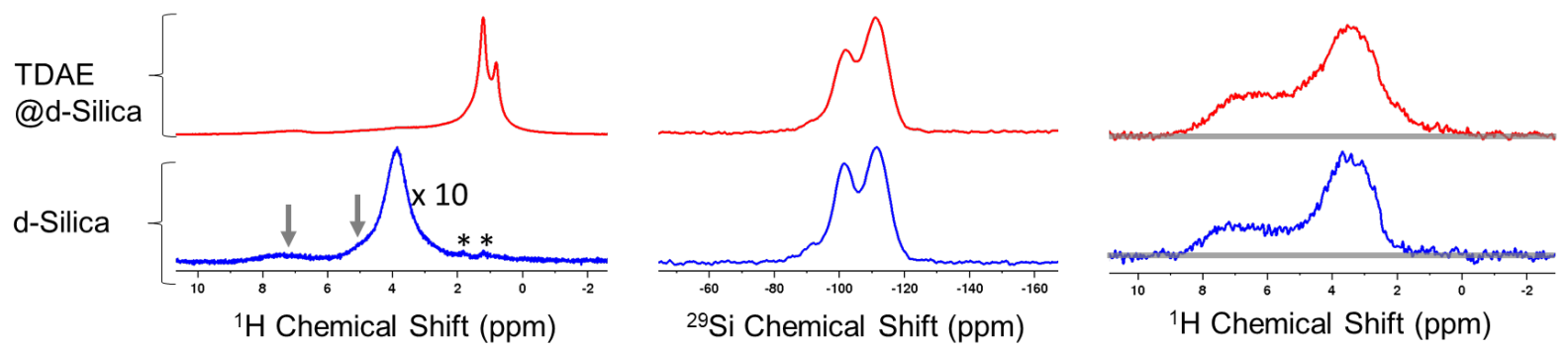

Figure S8. From left to right: ${ }^{1} \mathrm{H}$ MAS, $\left\{{ }^{1} \mathrm{H}-\right\}^{29} \mathrm{Si} \mathrm{MCP}$ and $\left\{{ }^{1} \mathrm{H}-{ }^{29} \mathrm{Si}-\right\}^{1} \mathrm{H} \mathrm{MCPi}$ spectra of bare and TDAE-adsorbed dsilicas. *Note: these two weak peaks are probably due to trace impurities. 


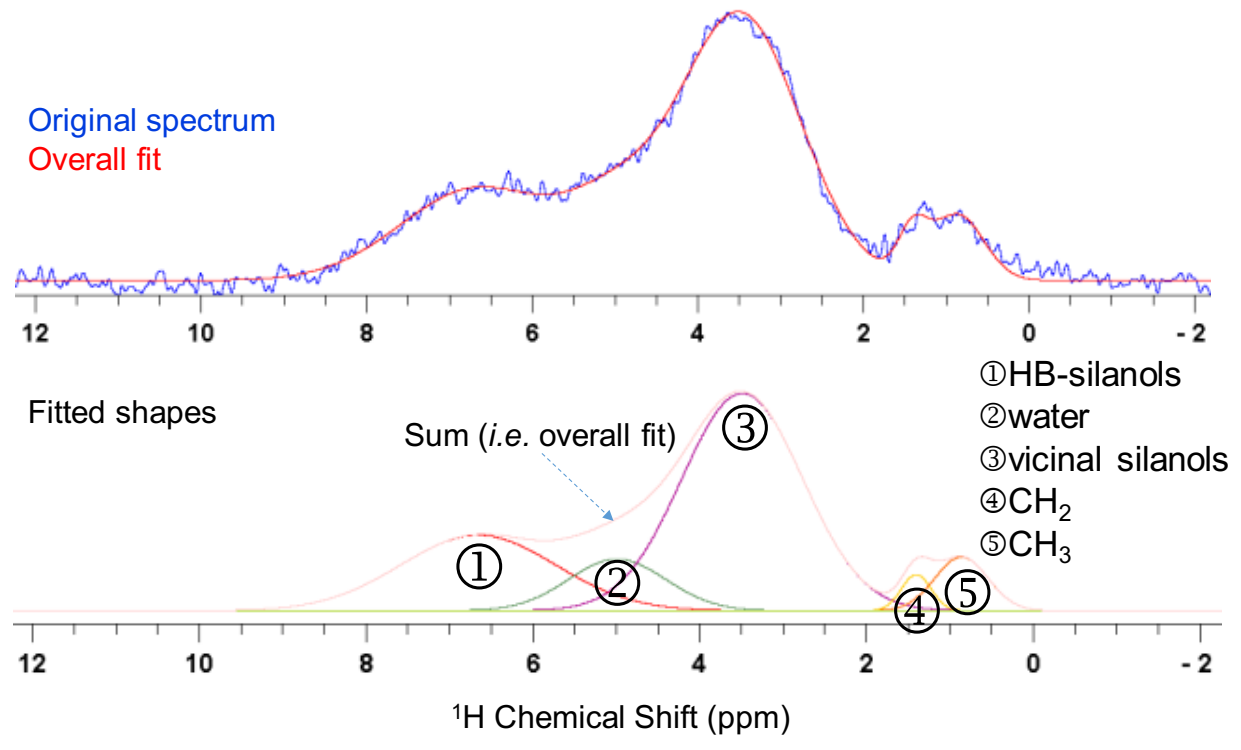

Figure S9. Deconvolution of the $\left\{{ }^{1} \mathrm{H}-{ }^{29} \mathrm{Si}-\right\}^{1} \mathrm{H}$ MCPi spectrum of SBO-E100@d-Silica.

Table S2. Calculations of ratios of the total region II to region I peak integrations as derived from the ${ }^{1} \mathrm{H}$ MCPi spectra shown in Figure 5, based on the deconvolution approach shown in Figure S9.

\begin{tabular}{|r|l|l|}
\hline${ }^{1} \mathrm{H}$ MCPi spectra of & $($ (4) + (5) / (1) + (2) + (3) & Estimated Error* \\
\hline d-Silica & $1585 / 146888=0.009$ & \pm 0.0015 \\
\hline TDAE@d-Silica & $4468 / 173234=0.026$ & \pm 0.0007 \\
\hline SBO@d-Silica & $4082 / 168373=0.024$ & \pm 0.0019 \\
\hline SBO-E20@d-Silica & $5876 / 168432=0.035$ & \pm 0.0005 \\
\hline SBO-E40@d-Silica & $7335 / 182310=0.040$ & \pm 0.0050 \\
\hline SBO-E50@d-Silica & $10506 / 159703=0.066$ & \pm 0.0013 \\
\hline SBO-E75@d-Silica & $13249 / 151762=0.087$ & \pm 0.0031 \\
\hline SBO-E100@d-Silica & $14654 / 160897=0.091$ & \pm 0.0017 \\
\hline
\end{tabular}

${ }^{*}$ Note: the estimated errors were calculated by error propagation of the errors from the deconvolution algorithm (Bruker command dconv). A gaussian function $\mathrm{g}(\mathrm{x})$ of integral $\mathbf{A}$ is described as

$$
g(x)=\frac{\boldsymbol{A}}{\sigma \sqrt{2 \pi}} e^{\frac{-x^{2}}{2 \sigma^{2}}}
$$

The factor $\frac{A}{\sigma \sqrt{2 \pi}}$ corresponds to the maximum height $\mathbf{i}$ and the full width at half maximum (FWHM) $\mathbf{w}$ can be shown to equal $2 \sqrt{2 \ln (2)} \sigma$.

Hence $\sigma$ and the area $\mathbf{a}$, as well as the corresponding errors $\delta \sigma$ and $\delta \mathbf{a}$ can be calculated using the following relations:

$$
\begin{array}{ll}
\sigma=\frac{\boldsymbol{w}}{2 \sqrt{(2 \ln 2)}} & \delta \sigma=\frac{\delta \boldsymbol{w}}{2 \sqrt{(2 \ln 2)}} \\
\boldsymbol{a}=i \cdot \sigma \sqrt{(2 \pi)} & \delta \boldsymbol{a}=\boldsymbol{a}\left(\frac{\delta \boldsymbol{w}}{\boldsymbol{w}}+\frac{\delta \boldsymbol{\sigma}}{\sigma}\right)
\end{array}
$$




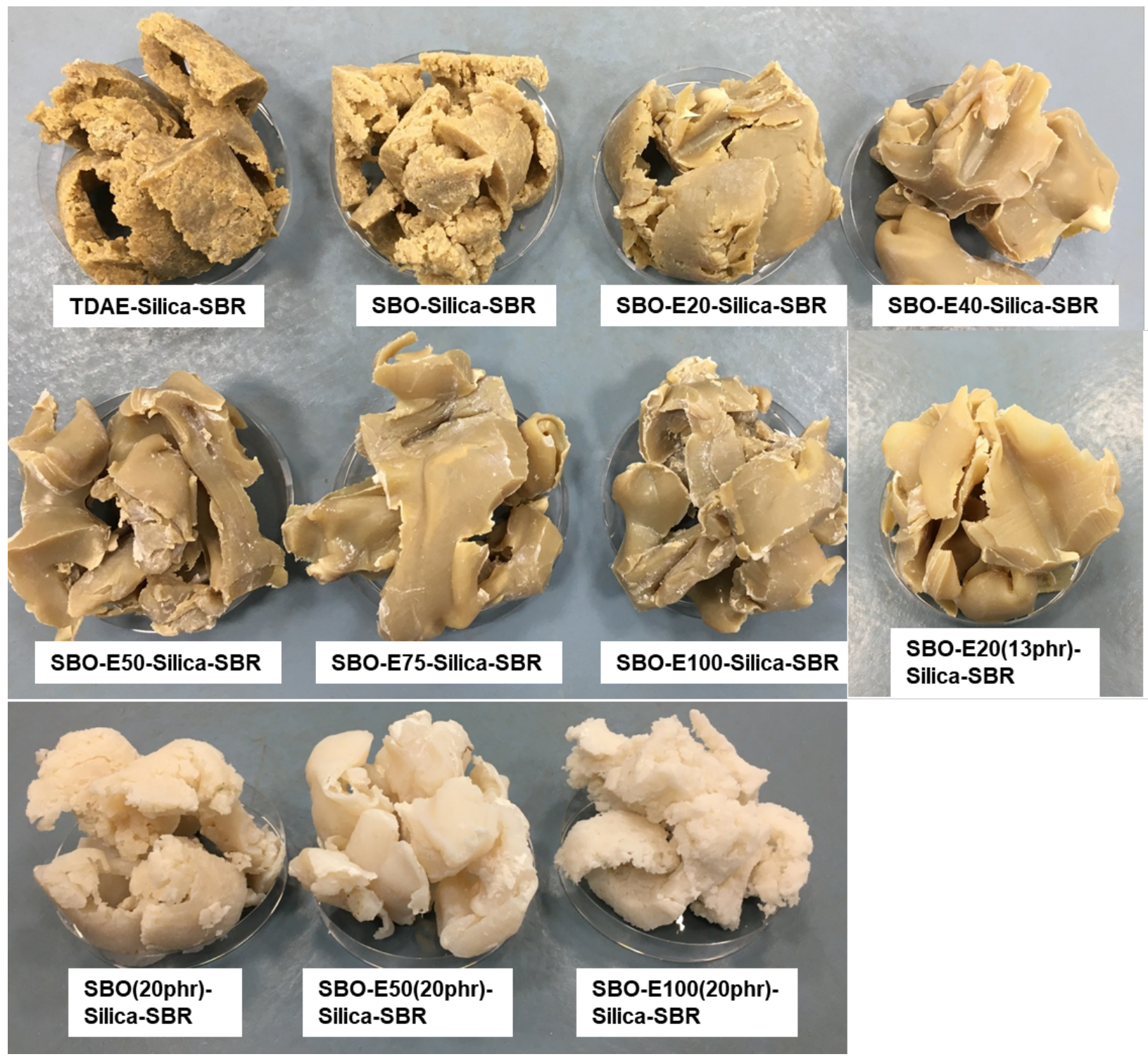

Figure S10. The physical appearance of the model rubber compounds. 


\section{Supporting Information}
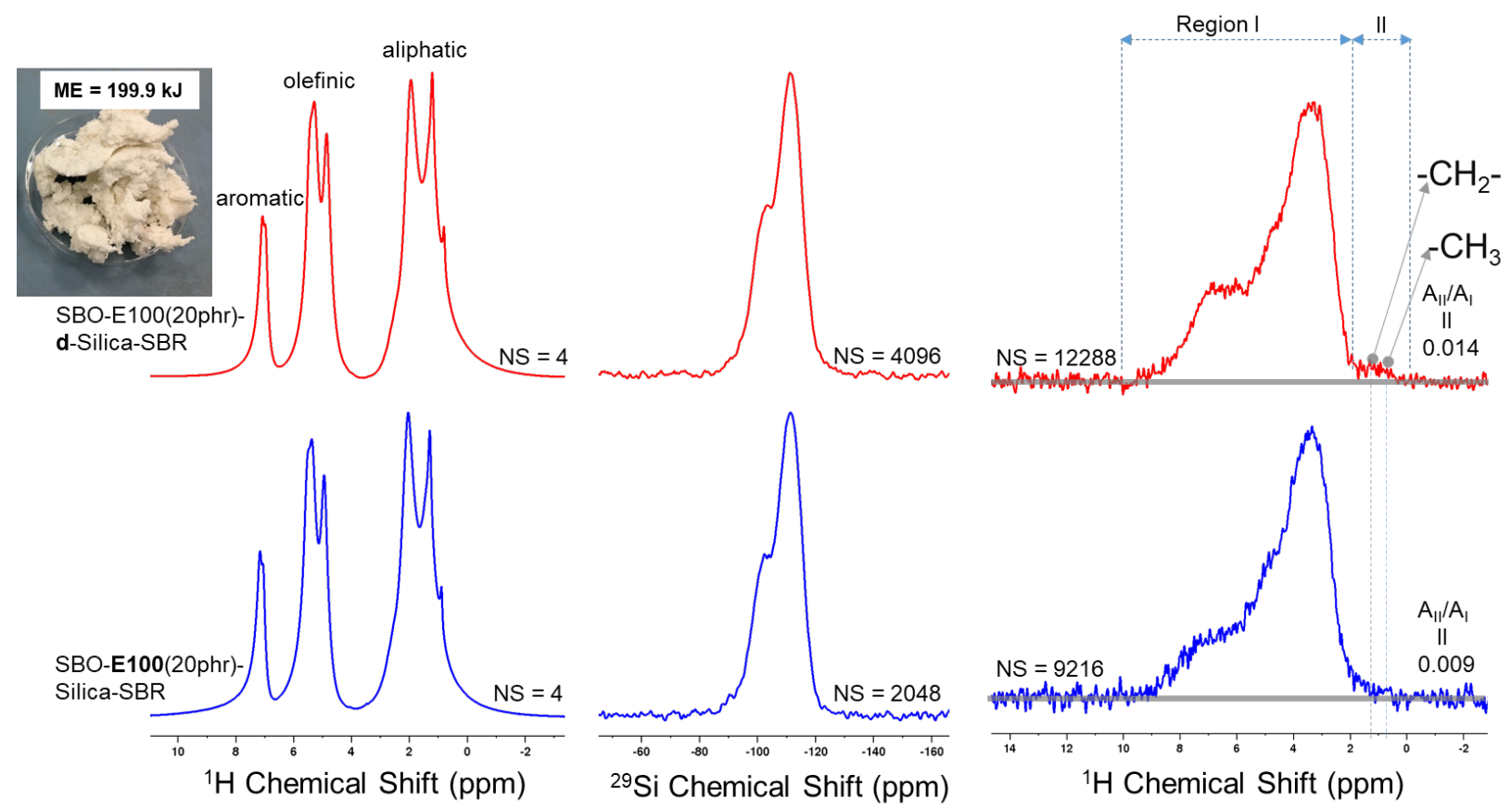

Figure S11. Left to right column: ${ }^{1} \mathrm{H}$ MAS, $\left\{{ }^{1} \mathrm{H}-\right\}^{29} \mathrm{Si} \mathrm{MCP}$ and $\left\{{ }^{1} \mathrm{H}-{ }^{29} \mathrm{Si}-\right\}^{1} \mathrm{H}$ MCPi spectra of two rubber compounds: SBO-E100(20phr)-d-Silica-SBR and SBO-E100(20phr)-Silica-SBR. Total number of scans (NS) of each spectrum was given, and the photo and the mixing energy (only one mixing) of SBO-E100(20phr)-d-Silica-SBR were also inserted on the top row. 